\title{
HOTAIR upregulates an 18-gene cell cycle-related mRNA network in glioma
}

\author{
KAI HUANG ${ }^{1,2^{*}}$, JIA SUN $^{2,3^{*}}$, CHAO YANG ${ }^{1,2^{*}}$, YUNFEI WANG ${ }^{1,2}$, BINGCONG ZHOU $^{1,2}$, \\ CHUNSHENG KANG $^{1,2}$, LEI HAN ${ }^{1,2}$ and QIXUE WANG ${ }^{1,2}$ \\ ${ }^{1}$ Department of Neurosurgery, Tianjin Medical University General Hospital; \\ ${ }^{2}$ Laboratory of Neuro-Oncology, Tianjin Neurological Institute, Tianjin 300052; ${ }^{3}$ ProteinT Biotec, \\ Tianjin Economic-Technological Development Area (TEDA), Tianjin 300457, P.R. China
}

Received November 9, 2016; Accepted February 24, 2017

DOI: $10.3892 /$ ijo.2017.3901

\begin{abstract}
HOTAIR is a tumor promoting long non-coding RNA (lncRNA) with roles in multiple cancers. However, the role of HOTAIR in glioma has not been well charaterized. Genes that positively correlated with HOTAIR were identified from the Chinese Glioma Genome Atlas and constructed into an interacting network. In total, 18 genes with P-values $<0.01$ were further extracted and constructed into a subnetwork. Realtime PCR, western blot and immunofluorescence analyses were employed to examine the expression of the genes after HOTAIR overexpression or knockdown. Intracranial glioblastoma multiform (GBM) models were used to test the potential of HOTAIR as a glioma therapy target. It was discovered that the 18 genes that most significantly correlated with HOTAIR expression formed a cell cycle-related mRNA network, which is positively regulated by HOTAIR. Furthermore, HOTAIR knockdown inhibited mouse intracranial GBM model formation. HOTAIR positively regulates a cell cycle-related mRNA network in glioma, and could be a potential therapeutic target for treating glioma.
\end{abstract}

\section{Introduction}

Gliomas are the most common primary brain tumors. The average survival rate of grade III patients is 3-5 years, whereas for GBM patients, the average survival is 12-15 months. Surgical resection, standard chemotherapy and radiation therapy provide little improvement in the outcomes of these

Correspondence to: Dr Lei Han or Dr Qixue Wang, Department of Neurosurgery, Tianjin Medical University General Hospital, 154 Anshan Road, Heping, Tianjin 300052, P.R. China

E-mail: superhanlei@hotmail.com

E-mail: qixue_wang@foxmail.com

*Contributed equally

Key words: HOTAIR, cell cycle, mRNA network, glioma patients (1). Thus, further exploration of the molecular mechanism may shed light on the treatment of glioma.

Long non-coding RNAs (lncRNAs) are a type of noncoding RNAs that are longer that $200 \mathrm{nt}$, and play various roles in diverse biological processes. They can affect expression of downstream genes, alter alternative splicing by hybridizing to pre-mRNA, are involved in structural and organizational roles in the cell and can be processed into small RNAs (2-4). However, the molecular mechanisms of lncRNA are not completely understood.

HOTAIR is an IncRNA that is much more characterized than other long non-coding transcripts. In developmental processes, HOTAIR silences the expression of the HOXD gene cluster (5). Targeted disruption of HOTAIR leads to gene de-repression and homeotic transformation in mice (6). Deletion of 5' HOXC genes where HOTAIR locates, leads to malformations in mice, such as clubfoot and vertical talus (7). HOTAIR was first reported as a tumor-promoting lncRNA in breast cancer (8). Increased research indicates that HOTAIR could be a potential biomarker and target in gastric cancer, colon cancer, cervical cancer and glioma (9-12). Mechanistically, the 5' domain of HOTAIR binds to the PRC2 complex, while the $3^{\prime}$ domain binds to the LSD1 complex. EZH2 is a major component of the PRC2 complex, which tri-methylates H3K 27 markers at the promoter of target genes. High levels of HOTAIR in cancer may inhibit tumor suppressor genes in epigenetic manner (13). Knocking down HOTAIR in glioma has been shown to upregulate NLK, a negative regulator of $\beta$-catenin pathway, which depends on the $5^{\prime}$ domain but not the $3^{\prime}$ domain (14). As a cell cycle-associated gene, HOTAIR is a strong predictor of survival in glioma, and is often expressed in classical and mesenchymal subtypes (15).

Accurate control of the cell cycle is essential for DNA synthesis and cell proliferation. Aberrant cell cycle progression is commonly observed in tumors and closely tracks with abnormal expression of cell cycle checkpoint genes. LncRNAs impact the cell cycle in various ways (16-18). $N c R N A_{C C N D I}$ is a transcriptional regulator that suppresses cyclin D1 and participates in G1 arrest in a DNA damage-dependent manner (19). ANRIL, an antisense lncRNA in the INK4 locus, suppresses p15 ${ }^{\text {INK4B }}$ expression in a PRC2-dependent manner (20). MALAT1, a nuclear-localized lncRNA, controls the cell cycle 
via regulating B-MYB (21). Previously, we reported HOTAIR as a cell cycle-associated lncRNA. Herein, we further characterized the regulation mechanisms of HOTAIR.

In the present study, we identified a cell cycle-related mRNA network that is regulated by HOTAIR in glioma cells. Genes in the CGGA database whose expression is positively correlated with HOTAIR were constructed into an interaction network, ranked by connection. The top 18 genes with P-values $<0.01$ are associated with the cell cycle. In glioma cell lines, HOTAIR upregulated the mRNA expression of network, whereas knocking down HOTAIR inhibited expression of these genes. Finally, we demonstrated that knocking down HOTAIR in U87vIII cells significantly inhibited intracranial tumor growth. These results support the potential of targeting HOTAIR in glioma, and further supported that HOTAIR is the potential therapy target in glioma.

\section{Materials and methods}

Datasets of glioma samples. mRNA expression datasets and the corresponding clinical information were downloaded from the following websites: Chinese Glioma Genome Atlas (CGGA) (http://www.cgga.org.cn), the Cancer Genome Atlas (TCGA) (http://cancergenome.nih.gov), and the REpository for Molecular BRAin Neoplasia DaTa (REMBRANDT) (http://caintegrator.nci.nih.gov/rembrandt/).

Cell line and culture conditions. The human GBM cell lines U87, U87vIII and U251 were used for experiments. U87 and U251 GBM cells were purchased from the ATCC (Manassas, VA, USA). U87 cells were cultured in complete MEM medium, while U251 cells were cultured in complete EMEM medium containing $10 \% \mathrm{FBS}$, and incubated at $37^{\circ} \mathrm{C}, 5 \% \mathrm{CO}_{2}$. U87vlll cells were stably transfected with a truncated mutant EGFR gene, which can be consistently activated without EGF stimulation. Puromycin $(100 \mathrm{ng} / \mathrm{ml})$ was added into U87vIII culture medium to maintain the stability of EGFRvIII.

Reverse transcription (RT)-PCR. To determine mRNA expression, after treatment, the glioma cells were lysed in TRIzol reagent (Sigma-Aldrich, St. Louis, MO, USA). The lysate was well mixed with chloroform and spun for $15 \mathrm{~min}$ at $13,000 \mathrm{xg}$ at $4^{\circ} \mathrm{C}$. The top aqueous phase, which contains RNA, was collected and mixed with propan-2-ol to precipitate the total RNA, which was used for real-time PCR analysis of mRNA. The cDNA was synthesized from $1 \mu \mathrm{g}$ of total RNA, using a reverse transcription kit purchased from Promega (Madison, WI, USA), following the manufacturer's protocol. Real-time PCR was performed using a SYBR Green Master Mix from Life Technologies (Carlsbad, CA, USA). Amplification was performed by the DNA Engine Opticon 2 Two-Color Real-time PCR detection system (Bio-Rad Laboratories, Hercules, CA, USA). Relative gene expression was analyzed by $2 \Delta \Delta-\mathrm{Cq}$ method (22). Gene specific primers: HOTAIR-F: ATAGGC AAATGTCAGAGGGTT, HOTAIR-R: TCTTAAATTGG GCTGGGTC; GAPDH-F: GGTGGTCTCCTCTGACT TCAACA, GAPDH-R: GTTGCTGTAGCCAAATTCGT TGT; CCNA2-F: CTCTACACAGTCACGGGACAAAG, CCNA2-R: CTGTGGTGCTTTGAGGTAGGTC; HMMR-F: GGCTGGGAAAAATGCAGAGGATG, HMMR-R: CCTTT
AGTGCTGACTTGGTCTGC; FoxM1-F: CCTTCTGGA CCATTCACCCC, FoxM1-R: TCACCGGGAACTGGATA GGT; NUSAP1-F: CTGACCAAGACTCCAGCCAGAA, NUSAP1-R: GAGTCTGCGTTGCCTCAGTTGT; ASPM-F: GAGACCTTGGTGGAATACCTGC, ASPM-R: ACGAAGA TCCAAAAGCCTTGCAC; CDC6-F: TCCACCAAAGCAA GGCAAGA, CDC6-R: CGATCTGGGACAGCTGTGTT; FANCI-F: GCAAGCTGATGTTCGACTCATGC, FANCI-R: AGGCAGCAGATCAGGTTTTGGC; NCAPG-F: GACGA ACAGGAGGTGTCAGACT, NCAPG-R: TGCTGCGGT TTTGGCTCGTCTT; DLGAP5-F: CTCGATCAGCTACT CAAGCAGC, DLGAP5-R: CAGGTCTTCCTTTACTTG GCACC; CHEK1-F: ATCAACTCATGGCAGGGGTG, CHEK1-R: TCCAGCGAGCATTGCAGTAA; CEP55-F: TCG ACCGTCAACATGTGCAGCA, CEP55-R: GGCTCTGTG ATGGCAAACTCATG; KIF4A-F: TGCGTGGTCAAGTTT CGGAGTC, KIF4A-R: GCTGTAGGTCAGCAATCTGAGC; HJURP-F: TGAGAATTTGGGGTGGAAGACT, HJURP-R: AGCGGAGTCACACGTACATC; PLK4-F: GACACCTCAG ACTGAAACCGTAC, PLK4-R: GTCCTTCTGCAAATC TGGATGGC; CCNB2-F: AGTTCCAGTTCAACCCACCAA, CCNB2-R: TTGCAGAGCAAGGCATCAGA; CENPE-F: GGAGAAAGATGACCTACAGAGGC, CENPE-R: AGTT CCTCTTCAGTTTCCAGGTG; NCAPH-F: GACGAACAG GAGGTGTCAGACT, NCAPH-R: TGCTGCGGTTTTGG CTCGTCTT; AURKB-F: CATCCCAACATCCTGCGTCT, AURKB-R: AGCTCTCCCTTGAGCCCTAA.

Western blot analysis. Protein lysates were prepared as follows: after treatment, cells were washed twice with cold PBS, scraped and lysed in ice-cold RIPA buffer (Solarbio, Beijing, China). Lysate was sonicated for 20 cycles, and microcentrifuged for $15 \mathrm{~min}$ at $4^{\circ} \mathrm{C}$. The supernatant was transferred to a new test tube and stored at $20^{\circ} \mathrm{C}$. The protein samples were resolved by SDS-PAGE and transferred onto PVDF membranes (Milipore, Darmstadt, Germany). The membranes were then incubated with the following antibodies: anti-FoxM1 (Cell Signaling Technology, Danvers, MA, USA), anti-CEP55 (Abcam, Cambridge, UK) and GAPDH (Proteintech, Wuhan, China). Antibody-labeled protein bands on the PVDF membranes were detected using a G:BOX F3 (Syngene, Cambridge, UK).

Immunofluorescence analysis. U87 cells were seeded onto the coverslips and transfected with negative control or LentiHOTAIR virus (GeneChem, Shanghai, China) for $48 \mathrm{~h}$. Then, they were fixed in $4 \%$ paraformaldehyde for $30 \mathrm{~min}$. The cells or sections were permeabilized with $0.1 \%$ Triton-X 100 for $10 \mathrm{~min}$, followed by blocking with $3 \%$ BSA for $1 \mathrm{~h}$ in RT. Immunofluorescence staining was conducted with antibodies against CEP55 (Abcam, 1:100) and CENPE (Abcam, 1:100). The cells were washed with PBS and incubated with Alexa Fluor 633 or Alexa Fluor 594 (Life Technologies) secondary antibodies. Nuclei were stained using DAPI and the cells were visualized using FV-1200 laser scanning confocal microscope.

Mouse glioma intracranial model and treatment. U87vIII cells were transfected with either a negative control or Lentisi-HOTAIR virus. An indicated number of cells in suspension were injected stereotactically into the brain of 4-week-old $\mathrm{BALB} / \mathrm{c}-\mathrm{nu}$ mice. The mice were sacrificed at day 14 . 


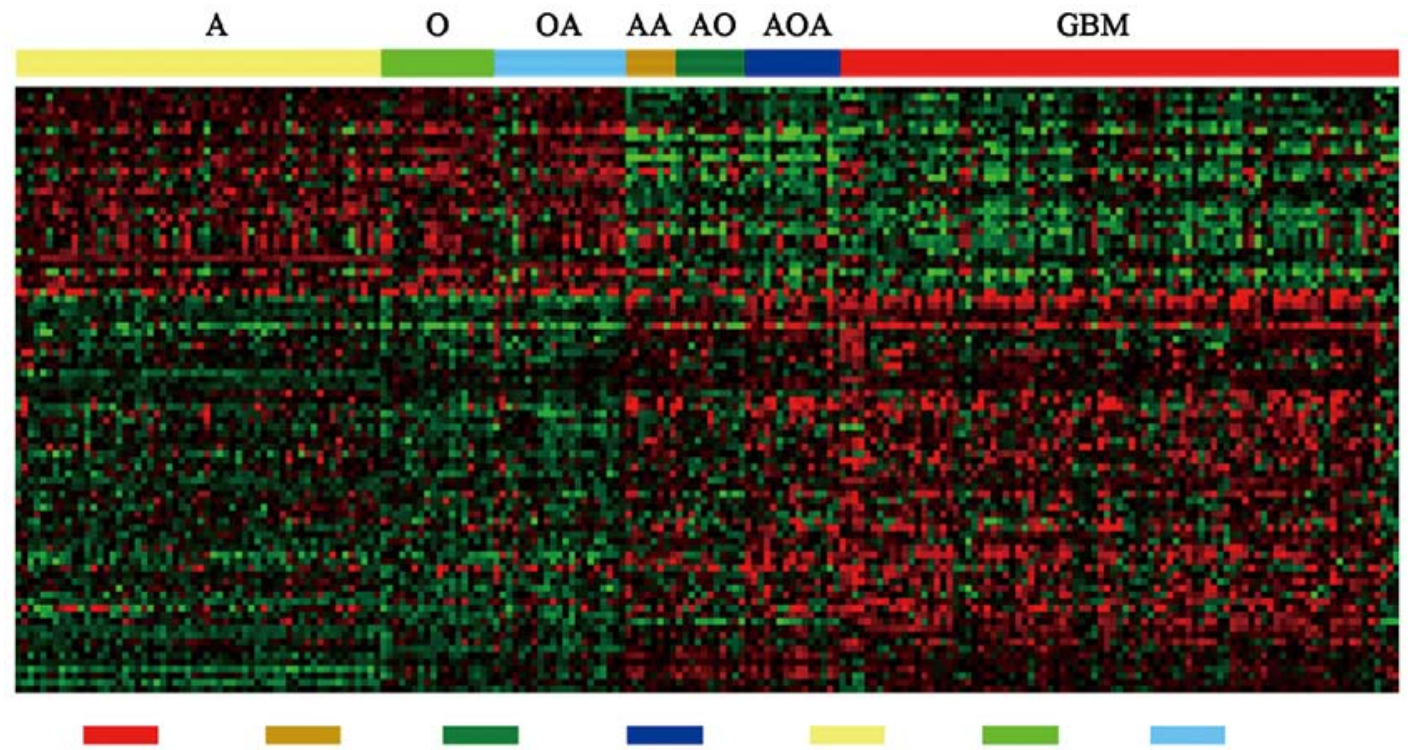

Figure 1. Differing patterns of lncRNA expression in glioma. The relative expression of lncRNAs in CGGA database was sorted by the clinical glioma grades.

A

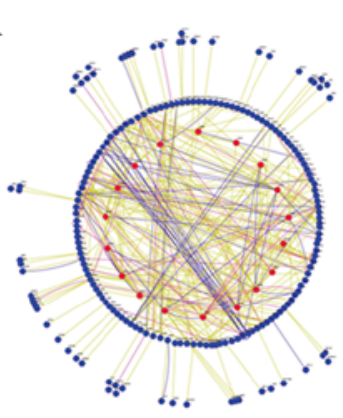

$\mathbf{F}$

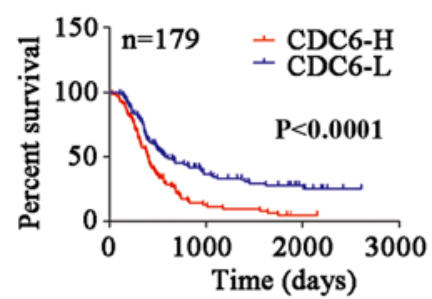

G

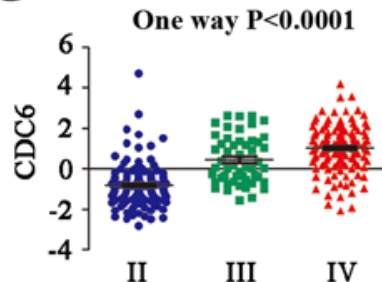

B

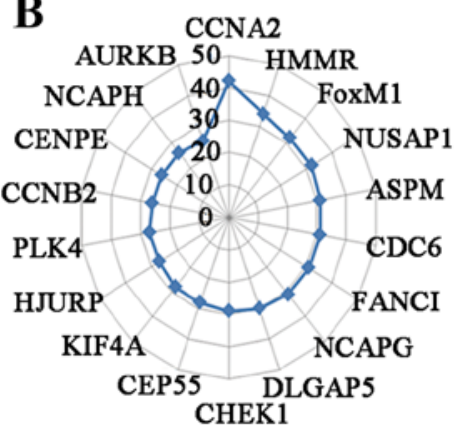

H

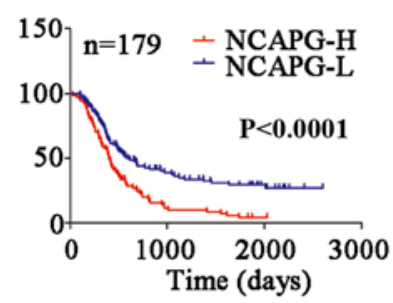

I

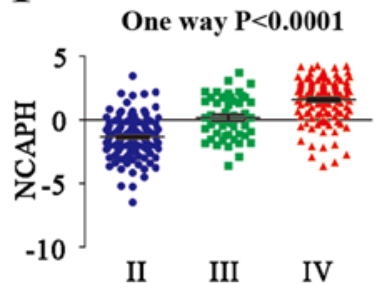

C

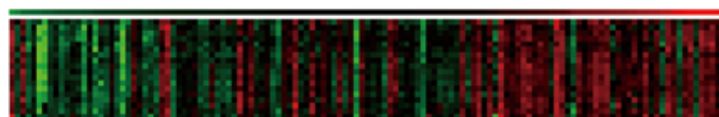

D

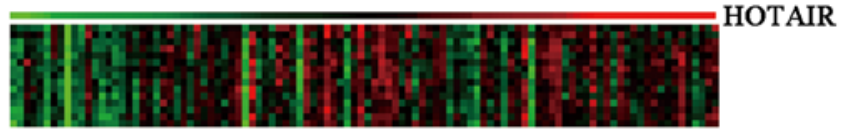

$\mathbf{E}$

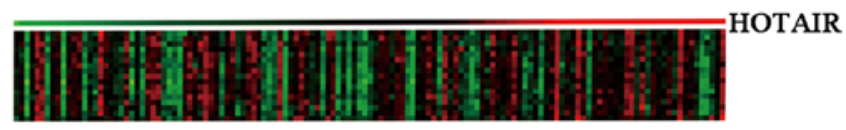

$\mathbf{J}$

$\mathbf{L}$

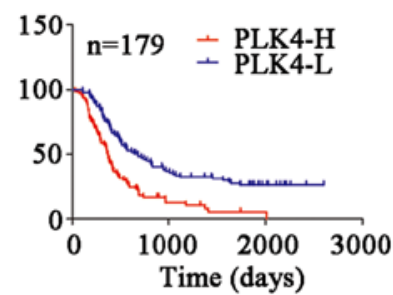

$\mathbf{K}$

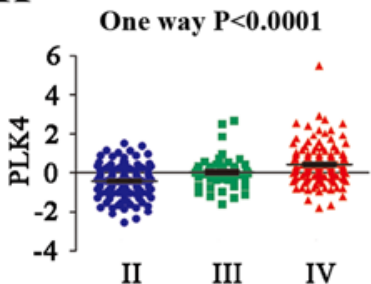

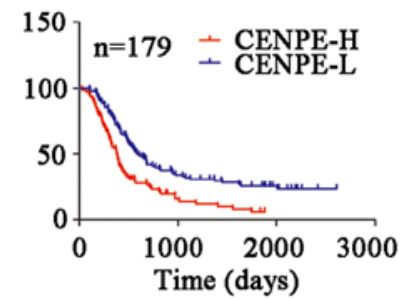

HOTAIR

\section{M}

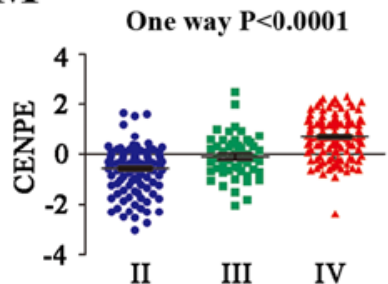

Figure 2. HOTAIR correlates with a cell cycle-related mRNA network. (A) Genes positively correlated with HOTAIR were constructed into an interacting network. (B) Genes with P-values $<0.01$ were selected and incorporated into a subnetwork based on connection. These 18 genes were clustered according to HOTAIR expression in the CGGA (C), TCGA (D) and REMBRANT databases (E). The expression of CDC6 (F), NCAPH (H), PLK4 (J) and CENPE (L) in different WHO grades was analyzed with the CGGA database. According to the expression value of CDC6 (G), NCAPH (I), PLK4 (K) and CENPE (M), patients with high-grade glioma could be divided into two groups with significantly different prognoses in the CGGA database. High group: patients with indicated gene expression higher than or equal to the median one. Low group: patients with lower indicated gene levels lower than the median one.

Histological analysis. The xenograft samples were collected at day 14 after tumor implantation, and were subjected to
Hematoxylin and eosin (H\&E) staining. Sections $(5 \mu \mathrm{m})$ were cut, dehydrated, deparaffinized, and rehydrated. 

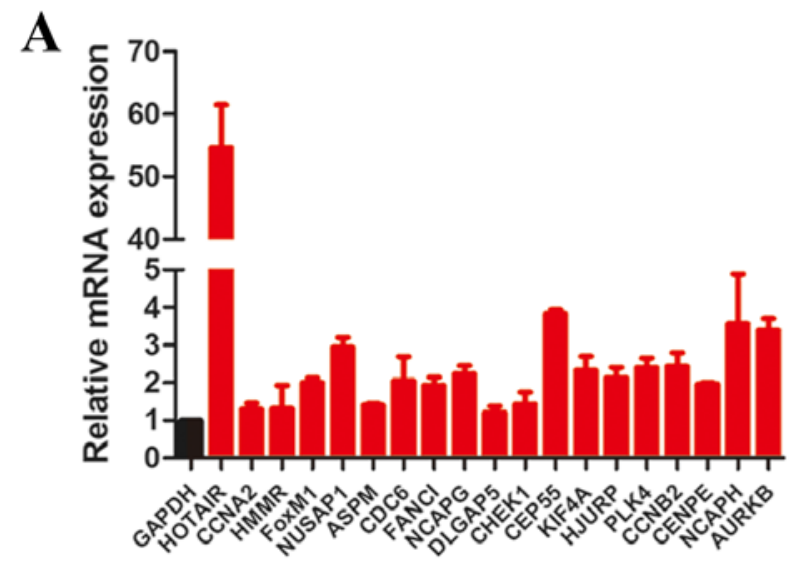

C

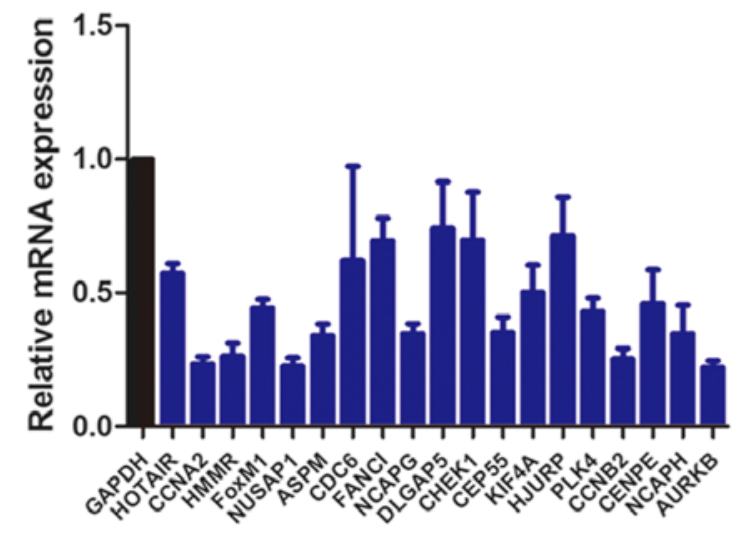

$\mathbf{E}$

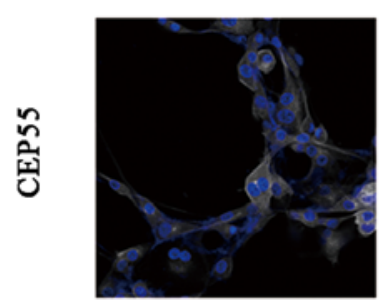

NC

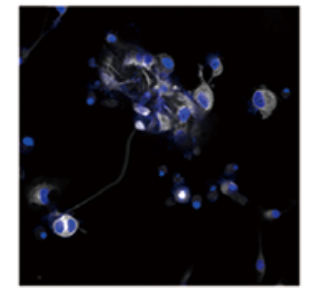

HOTAIR
B

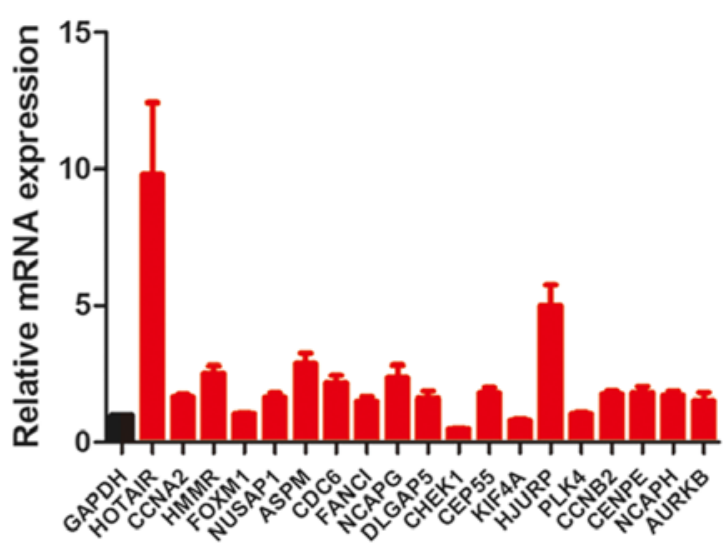

D

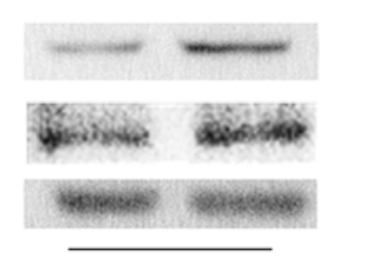

U87
NC si-HOTAIR

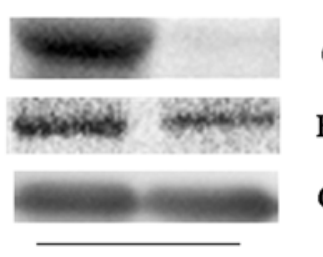

U87vIII

CEP55

FoxM1

GAPDH

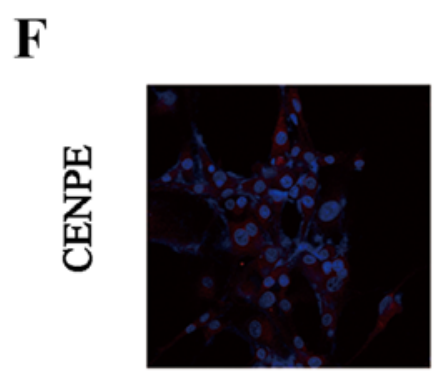

NC

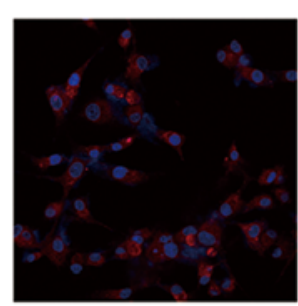

HOTAIR

Figure 3. HOTAIR positively regulates the cell cycle-related mRNA network. The expression of 18 genes in the cell cycle-related mRNA network was assessed by real-time PCR after HOTAIR overexpression in U87 (A) and U251 cells (B), and HOTAIR knockdown in U87vIII cells (C). (D) The expression of FoxM1 and CEP55 was examined by western blotting in U87 and U87vIII cells, with overexpression or knockdown of HOTAIR. CEP55 (E) and CENPE (F) were examined by immunofluorescence after HOTAIR overexpression in U87 cells.

H\&E staining was performed according to the standard protocols. All images were captured via microscopy (Olympus, Tokyo, Japan). The protocol for the animal study was approved by the Animal Ethics Committee of Tianjin Medical University.

Statistical analysis. Statistical analysis was performed using the SPSS Graduate Pack, version 11.0, statistical software (SPSS). Data are presented as means \pm SEM of three independent experiments or means \pm SD performed in triplicate. One-way ANOVA was used for comparison among the different groups. A P-value of 0.05 was considered to indicate a statistically significant difference.

\section{Results}

Identification of a cell cycle-related mRNA network positively correlated with HOTAIR. Although variety of IncRNAs have been described in recent years, the functions of IncRNA are still not completely characterized. To profile lncRNAs in glioma, cluster analysis was employed using the CGGA database, which includes 220 glioma samples and 5 normal brain samples. There are $90 \mathrm{lncRNAs}$ that have different patterns of expression between low-grade and high-grade gliomas. There are 31 lncRNAs downregulated in high-grade glioma, while 59 lncRNAs were upregulated (FDR $<0.05$, fold $>1.5$ ) (Fig. 1). It is possible that the main alteration of IncRNA expression 
A

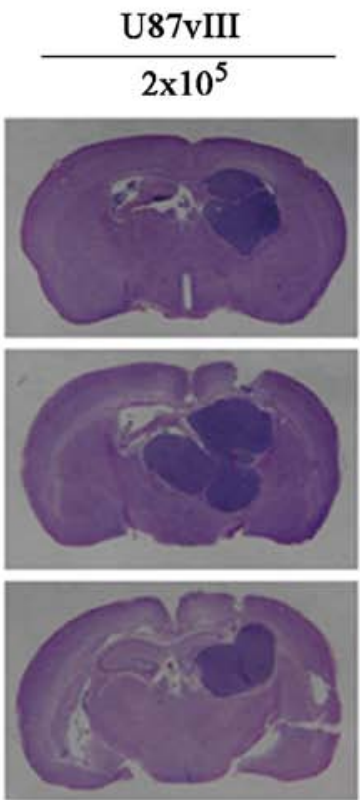

$3 / 3$
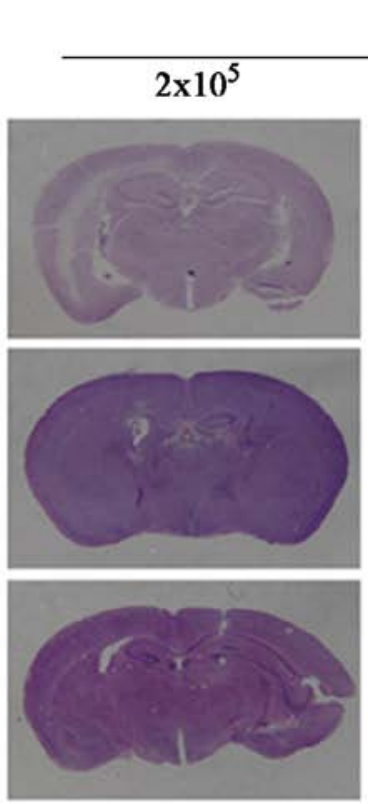

0/5
U87vIII-si-HOTAIR
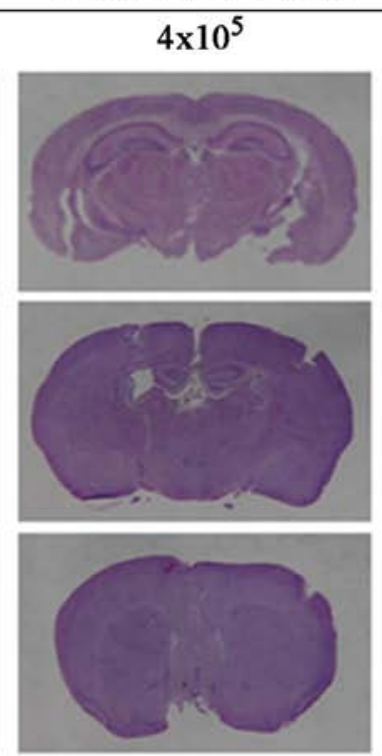

$0 / 5$
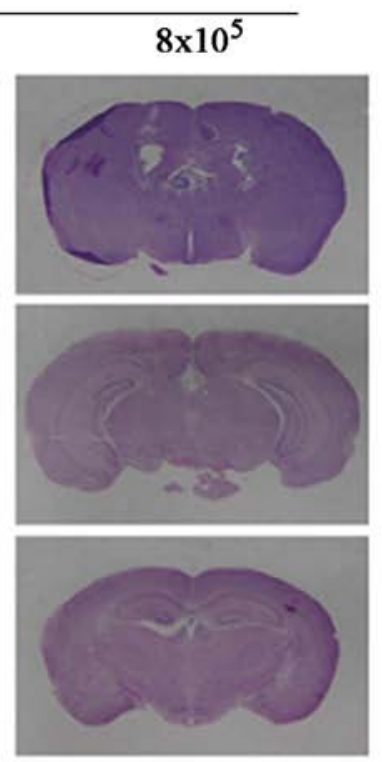

$0 / 4$
B

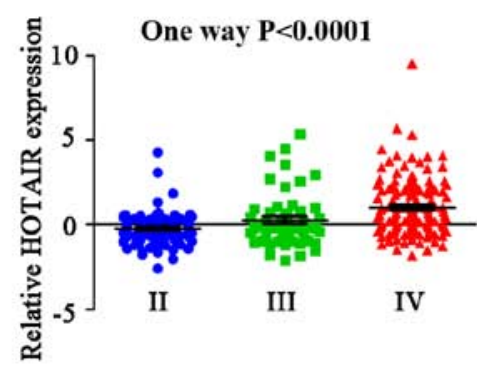

C

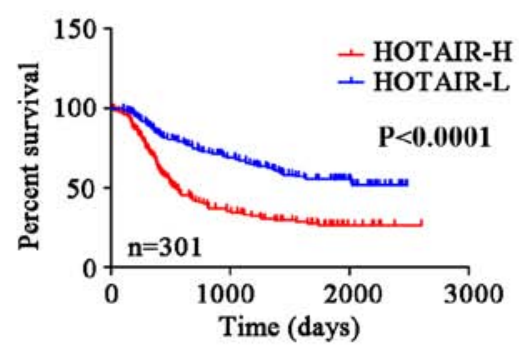

D

Figure 4. Targeting HOTAIR inhibits U87vIII intracranial glioma model. (A) H\&E staining of tissue from mice with U87vIII (negative control or Lenti-si HOTAIR treatment) on day 14 after implantation. (B) HOTAIR expression levels in WHO glioma grades were analyzed in the CGGA database. Kaplan-Meier survival curves for HOTAIR expression in glioma (C) and Grade III and IV (D).

occurs during malignant progression from low-grade glioma to GBM, indicating an important role of lncRNA in glioma progression. HOTAIR is among the lncRNAs in the high expressin module of the heat map, which is consistent with a previous study (15). HOTAIR is a cell cycle-associated oncogene in glioma (11), however, further research is still needed to demonstrate its role in gliomagenesis.

To profile the function of HOTAIR, genes positively correlated with HOTAIR were selected by Pearson correlation coefficients $(\mathrm{R}>0.3, \mathrm{P}<0.05)$ (Fig. 2A). In total, 244 HOTAIR correlated genes formed a complex network, in which they correlated with each other according to literature, databases or experiments. Genes with P-values $<0.01$ were extracted from the HOTAIR positive gene network, as they are more significantly correlated with HOTAIR (Fig. 2B). The correlation degrees of the 18 genes are greater than 25, and all of them interact with each other. Overall, 215 of the 244 genes in network are directly correlated with these 18 genes, implying a key role of this 18-gene network. The cluster analysis of these genes according to HOTAIR expression in the CGGA, REMBRANDT and TCGA databases further confirmed the positive correlation expression pattern of these 18 genes with HOTAIR (Fig. 2C-E). Bioinformatic analysis revealed that the upregulation of 17 of them (except CCNB2) corresponds with clinical stage glioma (Table I). Kaplan-Meier survival analysis further indicated a negative correlation between the expression level of 17 genes and the survival rate in highgrade glioma. Fig. 2F-M depicts four genes with the most significant P-values from the Kaplan-Meier survival analysis. Of these 18 genes, 15 genes play roles in cell cycle regulation, including CCNA2, FoxM1, CEP55, CENPE. FANCI and CDC6 are genes regulating DNA replication and repair, which may influence the cell cycle indirectly. Thus, we identified these mRNAs as a cell cycle-related network that positively correlated with HOTAIR.

HOTAIR upregulates a cell cycle-related mRNA network in vitro. To further test if this mRNA network could be upregulated by HOTAIR in vitro, we transfected a tetracyclineinducible HOTAIR expression lenti virus. Real-time PCR analysis indicated that genes in this network were upregulated when HOTAIR expression was induced by doxycycline in U87 (Fig. 3A) and U251 (Fig. 3B) cells. EGFRvIII is an aggressive EGFR mutation type in glioma. We knocked down HOTAIR in U87vIII cells by Lenti-si-HOTAIR and discovered that this network was downregulated by inhibiting HOTAIR (Fig. 3C). 
Table I. The cell cycle-related mRNA network that positively correlated with HOTAIR.

\begin{tabular}{|c|c|c|c|}
\hline Genes & Degrees & $\begin{array}{c}\text { Survival in HGG } \\
\text { (P-value) }\end{array}$ & UniProtKB/Swiss-Prot \\
\hline CCNA2 & 42 & 0.0004 & Controlling the cell cycle at the G1/S (start) and the G2/M (mitosis) transitions \\
\hline HMMR & 34 & 0.0018 & Phosphorylation of a number of proteins, including PTK2/FAK1 \\
\hline FoxM1 & 32 & 0.0032 & Regulates the expression of cell cycle genes essential for DNA replication and mitosis \\
\hline NUSAP1 & 32 & 0.0011 & Organization of mitotic spindle microtubules around them \\
\hline ASPM & 31 & 0.0002 & Mitotic spindle regulation and coordination of mitotic processes \\
\hline CDC6 & 31 & $<0.0001$ & Initiation of DNA replication \\
\hline FANCI & 31 & 0.0001 & Repairing of DNA double-strand breaks by homologous recombination \\
\hline NCAPG & 31 & $<0.0001$ & Conversion of interphase chromatin into mitotic-like condense chromosomes \\
\hline DLGAP5 & 30 & 0.0026 & Cell cycle regulator and key regulator of adherens junction integrity \\
\hline CHEK1 & 29 & 0.0059 & Binding to and phosphorylating CDC25A, CDC25B and CDC25C \\
\hline CEP55 & 28 & 0.0016 & Mitotic exit and cytokinesis \\
\hline KIF4A & 28 & 0.0117 & Mitotic chromosomal positioning and bipolar spindle stabilization \\
\hline HJURP & 27 & 0.0334 & Incorporation and maintenance of histone $\mathrm{H} 3$-like variant CENPA at centromeres \\
\hline PLK4 & 27 & $<0.0001$ & Central role in centriole duplication \\
\hline $\mathrm{CCNB} 2$ & 26 & 0.0646 & Controlling the cell cycle at the $\mathrm{G} 2 / \mathrm{M}$ (mitosis) transition \\
\hline CENPE & 26 & $<0.0001$ & Maintenance of chromosomal stability \\
\hline NCAPH & 26 & 0.0032 & Regulatory submit of the condensing complex \\
\hline AURKB & 25 & 0.0077 & Key regulator of mitosis \\
\hline
\end{tabular}

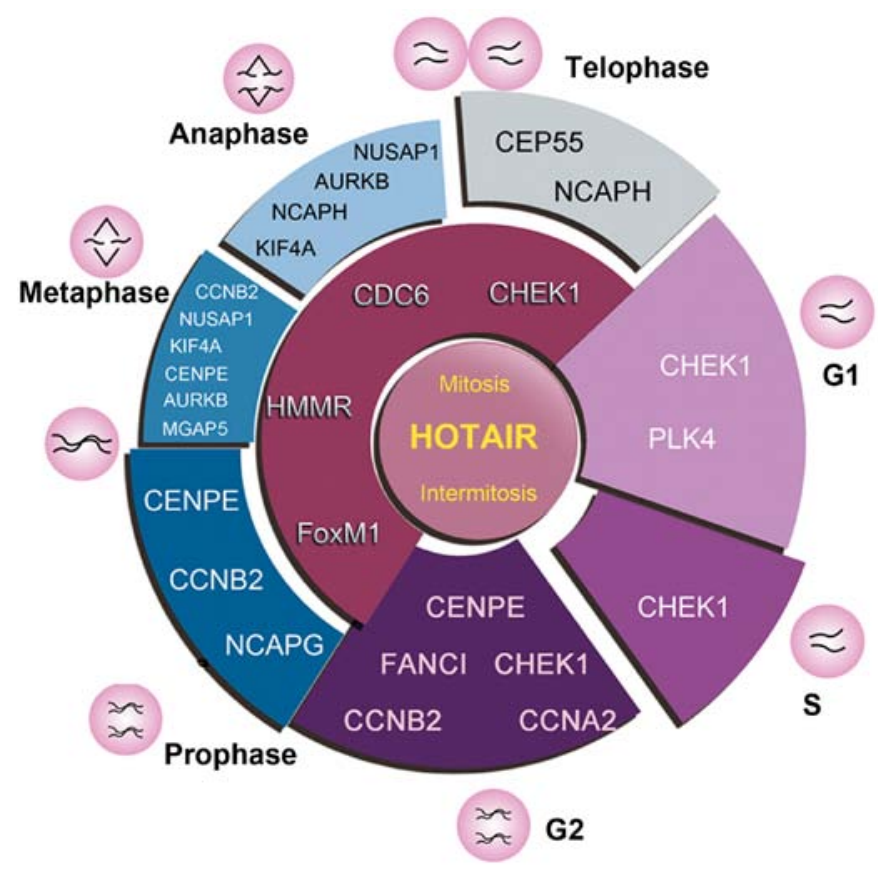

Figure 5. Genes in HOTAIR upregulated mRNA network are distributed throughout the cell cycle.

FoxM1 is a key transcription factor that regulates the expression of cell cycle genes $(23,24)$. CEP55 and CENPE, which are important regulators of mitosis, impact survival rate very significantly in high-grade glioma (HGG) $(24,25)$. Thus, we representatively chose FoxM1, CEP55 and CENPE from the network to further examine the influence of HOTAIR on these genes and the proteins they code. Western blot analysis indicated that HOTAIR could upregulate FoxM1 and CEP55 in U87 cells, while Lenti-si-HOTAIR-treatment inhibited expression of these proteins in U87vIII cells (Fig. 3D). Immunofluorescence staining further confirmed the upregulation of CEP55 (Fig. 3E) and CENPE (Fig. 3F) in U87 cells. Thus, we demonstrated that this cell cycle-related mRNA network is positively regulated by HOTAIR.

Targeting HOTAIR inhibits glioma progression. EGFR is a receptor tyrosine kinase that is frequently amplified and mutated in several cancers. EGFRvIII is one such mutation commonly found in GBM patients, and it contributes to the malignant progression of the disease (26). Knocking down HOTAIR significantly inhibited the cell cycle-related mRNA network in U87vIII cells. Thus, we targeted HOTAIR in U87vIII intracranial GBM mouse models. U87vIII cells $\left(2 \times 10^{5}\right)$ could form orthotopic tumors in mice in 14 days. However, after lenti-si-HOTAIR treatment, there was no macroscopic tumor formation even after the injection of $8 \times 10^{5}$ cells (Fig. 4A). These results indicated that knocking down HOTAIR could control U87vIII tumor formation within 2 weeks. Our bioinformatic analysis of the CGGA database further indicated that HOTAIR expression is upregulated in high-grade glioma (Fig. 4B), and high levels of HOTAIR are correlated with poor outcome in glioma (Fig. 4C) and HGG (Fig. 4D). These results indicated that HOTAIR could be a potential therapeutic target in glioma, especially for GBM.

\section{Discussion}

In the present study, we demonstrated that HOTAIR regulates a cell cycle-related mRNA network in glioma. Genes in this 
network are distributed throughout the cell cycle (Fig. 5). When leaving $G_{0}$ phase to enter into mitosis, healthy cells check the integrity of chromosomes and proteins required for replication. CCNA2, CCNB2 and CHEK1 promote this cell cycle checkpoint (27-29). During mitosis, the localization of centrosomes and spindles is well organized to perform accurate separation of the two daughter cells. NCAPH, CENPE, KIF4A, CEP55, NUSAP1 and ASPM participate in mitotic spindle regulation and mitotic processes (25,30-34). FoxM1 and AURKB are key regulators of mitosis (35-37). Thus, HOTAIR may regulate the cell cycle in glioma by impacting both checkpoint proteins and functional proteins during multiple mitosis steps.

Ten genes [HMMR (38), FoxM1 (39), ASPM (40), CDC6 (41), NCAPG (42), CHEK1 (43), CEP55 (44), HJURP (45), CENPE (42), ARUKB (46)] in this network are reported to promote glioma, through regulating the cell cycle and chemoresistance in glioma. Although the other genes have not yet been deeply studied in glioma, their expression is positively correlated with clinical grade glioma (Table I), which makes them potential therapeutic targets. Moreover, they participate in tumor progression in several cancers, including lung cancer and breast cancer (47-52). This evidence further confirmed the tumor-promoting role of HOTAIR in glioma and other cancers. Our results indicated that HOTAIR performs its carcinogenesis effort not only by inhibiting tumor-suppressor genes, but also by promoting expression of various oncogenes. This is the first report that HOTAIR could positively regulate a complex oncogene mRNA network, which further profiled the function of HOTAIR. However, the mechanism of upregulation of this network by HOTAIR remains to be explored. This upregulation could either be directly executed by HOTAIR, or be the cascading effort of network interactions.

In conclusion, we report for the first time that HOTAIR could positively regulate a complex oncogene mRNA network, which contributes to the further characterization of the functions of HOTAIR in glioma.

\section{Acknowledgements}

This study was supported by the National Key Research and Development Plan (2016YFC0902502), the China National Natural Scientific Fund (81572932), the Tianjin National Natural Scientific Fund (16JCYBJC27400) and the Tianjin Medical University General Hospital Incubation Fund (ZYYFY2014028).

\section{References}

1. Kalpathy-Cramer J, Gerstner ER, Emblem KE, Andronesi OC and Rosen B: Advanced magnetic resonance imaging of the physical processes in human glioblastoma. Cancer Res 74: 4622-4637, 2014.

2. Gloss BS and Dinger ME: The specificity of long noncoding RNA expression. Biochim Biophys Acta 1859: 16-22, 2016.

3. Yoon JH, Kim J and Gorospe M: Long noncoding RNA turnover. Biochimie 117: 15-21, 2015

4. St Laurent G, Wahlestedt C and Kapranov P: The Landscape of long noncoding RNA classification. Trends Genet 31: 239-251, 2015.

5. Rinn JL, Kertesz M, Wang JK, Squazzo SL, Xu X, Brugmann SA, Goodnough LH, Helms JA, Farnham PJ, Segal E, et al: Functional demarcation of active and silent chromatin domains in human HOX loci by noncoding RNAs. Cell 129: 1311-1323, 2007.
6. Li L, Liu B, Wapinski OL, Tsai MC, Qu K, Zhang J, Carlson JC Lin M, Fang F, Gupta RA, et al: Targeted disruption of Hotair leads to homeotic transformation and gene derepression. Cell Rep 5: 3-12, 2013.

7. Alvarado DM, McCall K, Hecht JT, Dobbs MB and Gurnett CA Deletions of $5^{\prime} \mathrm{HOXC}$ genes are associated with lower extremity malformations, including clubfoot and vertical talus. J Med Genet 53: 250-255, 2016.

8. Gupta RA, Shah N, Wang KC, Kim J, Horlings HM, Wong DJ, Tsai MC, Hung T, Argani P, Rinn JL, et al: Long non-coding RNA HOTAIR reprograms chromatin state to promote cancer metastasis. Nature 464: 1071-1076, 2010.

9. Jing L, Yuan W, Ruofan D, Jinjin Y and Haifeng Q: HOTAIR enhanced aggressive biological behaviors and induced radioresistance via inhibiting p21 in cervical cancer. Tumour Biol 36: 3611-3619, 2015.

10. Liu XH, Sun M, Nie FQ, Ge YB, Zhang EB, Yin DD, Kong R, Xia R, Lu KH, Li JH, et al: Lnc RNA HOTAIR functions as a competing endogenous RNA to regulate HER 2 expression by sponging miR-331-3p in gastric cancer. Mol Cancer 13: 92, 2014.

11. Zhang K, Sun X, Zhou X, Han L, Chen L, Shi Z, Zhang A, Ye M, Wang Q, Liu C, et al: Long non-coding RNA HOTAIR promotes glioblastoma cell cycle progression in an EZH2 dependent manner. Oncotarget 6: 537-546, 2015.

12. Kogo R, Shimamura T, Mimori K, Kawahara K, Imoto S, Sudo T, Tanaka F, Shibata K, Suzuki A, Komune S, et al: Long noncoding RNA HOTAIR regulates polycomb-dependent chromatin modification and is associated with poor prognosis in colorectal cancers. Cancer Res 71: 6320-6326, 2011.

13. Spitale RC, Tsai MC and Chang HY: RNA templating the epigenome: Long noncoding RNAs as molecular scaffolds. Epigenetics 6: 539-543, 2011.

14. Zhou X, Ren Y, Zhang J, Zhang C, Zhang K, Han L, Kong L, Wei J, Chen L, Yang J, et al: HOTAIR is a therapeutic target in glioblastoma. Oncotarget 6: 8353-8365, 2015.

15. Zhang JX, Han L, Bao ZS, Wang YY, Chen LY, Yan W, Yu SZ, Pu PY, Liu N, You YP, et al; Chinese Glioma Cooperative Group: HOTAIR, a cell cycle-associated long noncoding RNA and a strong predictor of survival, is preferentially expressed in classical and mesenchymal glioma. Neuro Oncol 15: 1595-1603, 2013.

16. McInerny CJ: Cell cycle regulated transcription: From yeast to cancer. F1000Res 5: 5, 2016.

17. Visconti R, Della Monica R and Grieco D: Cell cycle checkpoint in cancer: A therapeutically targetable double-edged sword. J Exp Clin Cancer Res 35: 153, 2016.

18. Bucher $\mathrm{N}$ and Britten CD: G2 checkpoint abrogation and checkpoint kinase-1 targeting in the treatment of cancer. $\mathrm{Br} \mathrm{J}$ Cancer 98: 523-528, 2008.

19. Wang X, Arai S, Song X, Reichart D, Du K, Pascual G, Tempst P, Rosenfeld MG, Glass CK and Kurokawa R: Induced ncRNAs allosterically modify RNA-binding proteins in cis to inhibit transcription. Nature 454: 126-130, 2008.

20. Kotake Y, Nakagawa T, Kitagawa K, Suzuki S, Liu N, Kitagawa M and Xiong Y: Long non-coding RNA ANRIL is required for the PRC2 recruitment to and silencing of p15 (INK4B) tumor suppressor gene. Oncogene 30: 1956-1962, 2011.

21. Tripathi V, Shen Z, Chakraborty A, Giri S, Freier SM, Wu X, Zhang Y, Gorospe M, Prasanth SG, Lal A, et al: Long noncoding RNA MALAT1 controls cell cycle progression by regulating the expression of oncogenic transcription factor B-MYB. PLoS Genet 9: e1003368, 2013.

22. Livak KJ and Schmittgen TD: Analysis of relative gene expression data using real-time quantitative PCR and the 2(-Delta Delta C(T)) method. Methods 25: 402-408, 2001.

23. Kwok CT, Leung MH, Qin J, Qin Y, Wang J, Lee YL and Yao KM: The Forkhead box transcription factor FOXM1 is required for the maintenance of cell proliferation and protection against oxidative stress in human embryonic stem cells. Stem Cell Res (Amst) 16: 651-661, 2016.

24. Barger CJ, Zhang W, Hillman J, Stablewski AB, Higgins MJ, Vanderhyden BC, Odunsi K and Karpf AR: Genetic determinants of FOXM1 overexpression in epithelial ovarian cancer and functional contribution to cell cycle progression. Oncotarget 6 : 27613-27627, 2015

25. Xu ZY, Ma XS, Qi ST, Wang ZB, Guo L, Schatten H, Sun QY and Sun YP: Cep55 regulates spindle organization and cell cycle progression in meiotic oocyte. Sci Rep 5: 16978, 2015. 
26. Zheng Q, Han L, Dong Y, Tian J, Huang W, Liu Z, Jia X, Jiang T, Zhang J, Li X, et al: JAK2/STAT3 targeted therapy suppresses tumor invasion via disruption of the EGFRvIII/JAK2/STAT3 axis and associated focal adhesion in EGFRvIII-expressing glioblastoma. Neuro Oncol 16: 1229-1243, 2014.

27. Loukil A, Cheung CT, Bendris N, Lemmers B, Peter M and Blanchard JM: Cyclin A2: At the crossroads of cell cycle and cell invasion. World J Biol Chem 6: 346-350, 2015.

28. Gong D and Ferrell JE Jr: The roles of cyclin A2, B1, and B2 in early and late mitotic events. Mol Biol Cell 21: 3149-3161, 2010.

29. Carrassa L and Damia G: Unleashing Chk1 in cancer therapy. Cell Cycle 10: 2121-2128, 2011.

30. Cabello OA, Eliseeva E, He WG, Youssoufian H, Plon SE, Brinkley BR and Belmont JW: Cell cycle-dependent expression and nucleolar localization of hCAP-H. Mol Biol Cell 12: 3527-3537, 2001

31. Vitre B, Gudimchuk N, Borda R, Kim Y, Heuser JE, Cleveland DW and Grishchuk EL: Kinetochore-microtubule attachment throughout mitosis potentiated by the elongated stalk of the kinetochore kinesin CENP-E. Mol Biol Cell 25: 2272-2281, 2014

32. Mazumdar M, Sundareshan S and Misteli T: Human chromokinesin KIF4A functions in chromosome condensation and segregation. J Cell Biol 166: 613-620, 2004.

33. Chou HY, Wang TH, Lee SC, Hsu PH, Tsai MD, Chang CL and Jeng YM: Phosphorylation of NuSAP by Cdk1 regulates its interaction with microtubules in mitosis. Cell Cycle 10: 4083-4089, 2011.

34. Higgins J, Midgley C, Bergh AM, Bell SM, Askham JM, Roberts E, Binns RK, Sharif SM, Bennett C, Glover DM, et al: Human ASPM participates in spindle organisation, spindle orientation and cytokinesis. BMC Cell Biol 11: 85, 2010.

35. Goldenson B and Crispino JD: The aurora kinases in cell cycle and leukemia. Oncogene 34: 537-545, 2015.

36. Nunes Bastos R, Gandhi SR, Baron RD, Gruneberg U, Nigg EA and Barr FA: Aurora B suppresses microtubule dynamics and limits central spindle size by locally activating KIF4A. J Cell Biol 202: 605-621, 2013.

37. Wierstra I: The transcription factor FOXM1 (Forkhead box M1): Proliferation-specific expression, transcription factor function, target genes, mouse models, and normal biological roles. Adv Cancer Res 118: 97-398, 2013.

38. Tilghman J, Wu H, Sang Y, Shi X, Guerrero-Cazares H, Quinones-Hinojosa A, Eberhart CG, Laterra J and Ying $M$ HMMR maintains the stemness and tumorigenicity of glioblastoma stem-like cells. Cancer Res 74: 3168-3179, 2014.

39. Zhang N, Wei P, Gong A, Chiu WT, Lee HT, Colman H, Huang H, Xue J, Liu M, Wang Y, et al: FoxM1 promotes $\beta$-catenin nuclear localization and controls Wnt target-gene expression and glioma tumorigenesis. Cancer Cell 20: 427-442, 2011.

40. Horvath S, Zhang B, Carlson M, Lu KV, Zhu S, Felciano RM, Laurance MF, Zhao W, Qi S, Chen Z, et al: Analysis of oncogenic signaling networks in glioblastoma identifies ASPM as a molecular target. Proc Natl Acad Sci USA 103: 17402-17407, 2006.
41. Stangeland B, Mughal AA, Grieg Z, Sandberg CJ, Joel M, Nygård S, Meling T, Murrell W, Vik Mo EO and Langmoen IA: Combined expressional analysis, bioinformatics and targeted proteomics identify new potential therapeutic targets in glioblastoma stem cells. Oncotarget 6: 26192-26215, 2015

42. Liang ML,Hsieh TH, Ng KH, Tsai YN, Tsai CF, Chao ME, Liu DJ, Chu SS, Chen W, Liu YR, et al: Downregulation of miR-137 and miR-6500-3p promotes cell proliferation in pediatric high-grade gliomas. Oncotarget 7: 19723-19737, 2016.

43. Tang Y, Dai Y, Grant S and Dent P: Enhancing CHK1 inhibitor lethality in glioblastoma. Cancer Biol Ther 13: 379-388, 2012.

44. Wang G, Liu M, Wang H, Yu S, Jiang Z, Sun J, Han K, Shen J, Zhu M, Lin Z, et al: Centrosomal Protein of 55 regulates glucose metabolism, proliferation and apoptosis of glioma cells via the Akt/mTOR signaling pathway. J Cancer 7: 1431-1440, 2016.

45. Valente V, Serafim RB, de Oliveira LC, Adorni FS, Torrieri R, Tirapelli DP, Espreafico EM, Oba-Shinjo SM, Marie SK, Paçó-Larson ML, et al: Modulation of HJURP (Holliday JunctionRecognizing Protein) levels is correlated with glioblastoma cells survival. PLoS One 8: e62200, 2013.

46. Diaz RJ, Golbourn B, Shekarforoush M, Smith CA and Rutka JT: Aurora kinase $\mathrm{B} / \mathrm{C}$ inhibition impairs malignant glioma growth in vivo. J Neurooncol 108: 349-360, 2012.

47. Takashima S, Saito H, Takahashi N, Imai K, Kudo S, Atari M, Saito Y, Motoyama S and Minamiya Y: Strong expression of cyclin B2 mRNA correlates with a poor prognosis in patients with non-small cell lung cancer. Tumour Biol 35: 4257-4265, 2014.

48. Shubbar E, Kovács A, Hajizadeh S, Parris TZ, Nemes S, Gunnarsdóttir K, Einbeigi Z, Karlsson P and Helou K: Elevated cyclin B2 expression in invasive breast carcinoma is associated with unfavorable clinical outcome. BMC Cancer 13: 1, 2013.

49. Kim DH, Park SE, Kim M, Ji YI, Kang MY, Jung EH, Ko E, Kim Y, Kim S, Shim YM, et al: A functional single nucleotide polymorphism at the promoter region of cyclin A2 is associated with increased risk of colon, liver, and lung cancers. Cancer 117: 4080-4091, 2011.

50. Moore NL, Edwards DP and Weigel NL: Cyclin A2 and its associated kinase activity are required for optimal induction of progesterone receptor target genes in breast cancer cells. J Steroid Biochem Mol Biol 144: 471-482, 2014.

51. Taniwaki M, Takano A, Ishikawa N, Yasui W, Inai $K$, Nishimura H, Tsuchiya E, Kohno N, Nakamura Y and Daigo Y: Activation of KIF4A as a prognostic biomarker and therapeutic target for lung cancer. Clin Cancer Res 13 (22 Pt 1): 6624-6631, 2007.

52. Wang H, Lu C, Li Q, Xie J, Chen T, Tan Y, Wu C and Jiang J: The role of Kif4A in doxorubicin-induced apoptosis in breast cancer cells. Mol Cells 37: 812-818, 2014. 